https://doi.org/10.15407/ujpe64.8.754

V. SKALOZUB

Oles Honchar Dnipro National University

(72, Gagarin Ave., Dnipro 49010,Ukraine; e-mail: Skalozubv@daad-alumni.de)

\title{
INDUCED COLOR CHARGES, EFFECTIVE $\gamma \gamma G$ VERTEX IN QGP. APPLICATIONS TO HEAVY-ION COLLISIONS
}

\begin{abstract}
We calculate the induced color charges $Q_{\text {ind }}^{3}, Q_{\text {ind }}^{8}$ and the effective vertex $\gamma-\gamma$-gluon generated in a quark-gluon plasma with the $A_{0}$ condensate because of the color $C$-parity violation at this background. To imitate the case of heavy-ion collisions, we consider the model of the plasma confined in the narrow infinite plate and derive the classical gluon potentials $\bar{\phi}^{3}$ and $\bar{\phi}^{8}$ produced by these charges. Two applications - the scattering of photons on a plasma and the conversion of gluon fields in two photons radiated from the plasma - are discussed.

Ke ywords: quark-gluon plasma, heavy-ion collision, Polyakov's loop, effective vertex.
\end{abstract}

\section{Introduction}

Investigations of the deconfinement phase transition (DPT) and the quark-gluon plasma (QGP) are in the center of modern high energy physics. These phenomena happen at high temperature due to the asymptotic freedom of strong interactions. The researches are carried out either in experiments on hadron collisions or in quantum field theory. The order parameter for DPT is Polyakov's loop (PL), which is zero at low temperatures and nonzero at high temperatures $T>T_{d}$, where $T_{d} \sim 160-180 \mathrm{MeV}$ [1] is the phase transition temperature. The standard information on DPT is adduced, in particular, in [2].

The PL is defined as [3]:

$P L=\int_{C} d x_{4} A_{0}\left(x_{4}, \mathbf{x}\right)$.

Here, $A_{0}\left(x_{4}, \mathbf{x}\right)$ is the zero component of the gauge field potential, the integration contour is going along the fourth direction and back to an initial point in the lattice Euclidean space-time. The PL was introduced in pure gluodynamics. It violates the center of the color group symmetry $Z(3)$ that results in the nonconservation of the color charges $Q^{3}$ and $Q^{8}$.

The QGP state consists of quarks and gluons liberated from hadrons. Polyakov's loop is not a solution to the local Yang-Mills equations. The local order parameter for DPT is the $A_{0}$ condensate, which

(c) V. SKALOZUB, 2019 is a constant at $T>T_{d}$. It can be calculated, in particular, from a two-loop effective potential. More details on different calculations carried out in analytic quantum field theory can be seen in [4]. Taking these results into consideration, we have to consider QGP as a state at the $A_{0}$ background, which breaks the color $C$-parity symmetry. So, new type phenomena may happen.

In the $\mathrm{SU}(2)$ gluodynamics, the gluon spectra at $A_{0}$ were calculated and investigated in Ref. [5, 6]. In particular, the induced color charge $Q_{\text {ind }}^{3}$ was also computed. It was shown that the state with a condensate is free of infrared instabilities existing in a gluon plasma in the empty space. Thus, the ground state with $A_{0}$ is a good approximation to the plasma after DPT.

In Ref. [7], the induced charges $Q_{\text {ind }}^{3}, Q_{\text {ind }}^{8}$ generated by quark loops in QCD were calculated. In what follows, we consider the QCD case, but the precise values of the induced charges will not be specified.

The paper is organized as follows. In Sect. 2, the color induced charges $Q_{\text {ind }}^{3}$ and $Q_{\text {ind }}^{8}$ generated by tadpole quark loops with one gluon lines, which are nonzero due to Furry's theorem violation, are calculated. In Sect. 3, we consider a simple model of the plasma confined in a plate narrow in one dimension and infinite in two other dimensions with the $A_{0}$ condensate and induced charges. We compute the classical gluon potentials $\bar{\phi}^{3}$ and $\bar{\phi}^{8}$ generated by the induced charges $Q_{\text {ind }}^{3}$ and $Q_{\text {ind }}^{8}$. In Sect. 4 , the effective $\gamma \gamma G$ vertex generated in the plasma is calculated in

ISSN 2071-0194. Ukr. J. Phys. 2019. Vol. 64, No. 8 
the high-temperature approximation. In Sect. 5, the processes of photon scattering on these potentials and the conversion of gluons in two photons are considered as the application. These new phenomena have to happen due to the three-linear effective vertices.

\section{Induced Color}

\section{Charges and Quark Propagator}

In what follows, we consider the case of $A_{0}^{3}$ background field and present the color field potential in the form $Q_{\mu}^{a} \rightarrow A_{0} \delta^{a 3} \delta_{\mu 4}+Q_{\mu}^{a}$, where $Q_{\mu}^{a}$ is a quantum field. The calculation of $Q_{\text {ind }}^{8}$ is similar (see [7]), and the final results will be adduced only.

The explicit expression is given by the form $Q_{\mu}^{a} Q_{\text {ind }}^{3} \delta_{\mu 4} \delta_{a 3}=Q_{4}^{3} Q_{\text {ind }}^{3}$, where

$Q_{\text {ind }}^{3}=\frac{g}{\beta} \sum_{p_{4}} \int \frac{d^{3} p}{(2 \pi)^{3}} \operatorname{Tr}\left[\gamma^{4} \frac{\lambda_{i j}^{3}}{2} G^{i j}\left(p_{4}, \mathbf{p}, A_{0}\right)\right]$.

Here, $\lambda^{3}$ is the Gell-Mann matrix, and $\beta=1 / T$ is the inverse temperature. The expressions for the propagators are

$$
\begin{aligned}
G^{11} & =\frac{\gamma^{4}\left(p_{4}-A_{0}\right)+\mathbf{p} \gamma+m}{\left(p_{4}-A_{0}\right)^{2}+\mathbf{p}^{2}+m^{2}}, \\
G^{22} & =\frac{\gamma^{4}\left(p_{4}+A_{0}\right)+\mathbf{p} \gamma+m}{\left(p_{4}+A_{0}\right)^{2}+\mathbf{p}^{2}+m^{2}} .
\end{aligned}
$$

For brevity, we denoted $A_{0}=g A_{0} / 2$ entering the interaction Lagrangian. Accounting for the trace $\operatorname{Tr}\left[\left(\gamma^{4}\right)^{2}\right]=-4$, the diagonal values of $\lambda^{3}$, and $\operatorname{Tr}\left[\gamma^{4} \gamma\right]=0$, we get

$Q_{\text {ind }}^{3}=\frac{4 g}{\beta} \sum_{p_{4}} \int \frac{d^{3} p}{(2 \pi)^{3}} \frac{p_{4}+A_{0}}{\left(p_{4}+A_{0}\right)^{2}+\mathbf{p}^{2}+m^{2}}$.

The sum over $p_{4}=\frac{\pi(2 n+1)}{2 \beta}$ can be calculated, by using the formula

$\frac{1}{\beta} \sum_{p_{4}} f\left(p_{4}\right)=-\frac{1}{4 \pi i} \int_{C} \tan \left[\frac{\beta \omega}{2}\right] f(\omega)$,

where the contour $C$ encloses clockwise the real axis in the complex plane $\omega$.

The calculations (after transformation to the spherical coordinates and angular integrations) give

$Q_{\text {ind }}^{3}=\frac{g \sin \left(A_{0} \beta\right)}{\pi^{2}} \int_{0}^{\infty} p^{2} d p \frac{1}{\cos \left(A_{0} \beta\right)+\cosh \left(\epsilon_{p} \beta\right)}$,

where $\epsilon_{p}^{2}=p^{2}+m^{2}$.

ISSN 2071-0194. Ukr. J. Phys. 2019. Vol. 64, No. 8
Considering the high-temperature limit $\beta \rightarrow \infty$, we obtain

$Q_{\text {ind }}^{3}=g A_{0}\left[\frac{4}{3} \beta^{-2}-\frac{2 m^{2}}{3 \pi^{2}} \beta+O\left(\beta^{3}\right)\right]$.

Hence, we see that the first term is independent of the mass and dominant at high temperatures.

Now, for completeness, we calculate the temperature sum in Eq. (4).

The integrand in Eq. (4) has the form

$f\left(p_{4}\right)=\frac{p_{4}+A_{0}}{\left(p_{4}-p_{4}^{(1)}\right)\left(p_{4}-p_{4}^{(2)}\right)}$,

where $p_{4}^{(1)}=-A_{0}+i \epsilon_{p}, p_{4}^{(2)}=-A_{0}-i \epsilon_{p}$. The sum in Eq. (5) after computing the simple residues equals

$$
\begin{aligned}
& S_{1}=\frac{1}{\beta} \sum_{p_{4}} f\left(p_{4}\right)=-\frac{1}{2}\left[\frac{i \epsilon_{p}}{p_{4}^{(1)}-p_{4}^{(2)}} \tan \left(\frac{\beta}{2} p_{4}^{(1)}\right)+\right. \\
& \left.+\frac{-i \epsilon_{p}}{p_{4}^{(2)}-p_{4}^{(1)}} \tan \left(\frac{\beta}{2} p_{4}^{(2)}\right)\right] .
\end{aligned}
$$

Substituting the corresponding parameters and fulfilling elementary transformations, we find

$S_{1}=\frac{1}{2} \frac{\sin \left(A_{0} \beta\right)}{\cos \left(A_{0} \beta\right)+\cosh \left(\epsilon_{p} \beta\right)}$.

By substituting $S_{1}$ in Eq. (4), we obtain Eq. (6).

Performing similar calculations for $Q_{\text {ind }}^{8}$, we get [7]

$Q_{\text {ind }}^{8}=g A_{0}^{8}\left[\frac{16}{3 \sqrt{3}} \beta^{-2}-\frac{8 m^{2}}{3 \sqrt{3} \pi^{2}} \beta+O\left(\beta^{3}\right)\right]$.

Here, $A_{0}^{8}$ is the background field generated in the plasma. For our problem, it is a given number.

Now, we calculate the quark propagator accounting for the induced charge by means of SchwingerDyson's equation. In the Euclidean space-time, it reads

$S^{-1}(p)=-\left(\gamma^{4}\left(p_{4}-\frac{\lambda^{3}}{2} g A_{0}\right)+\gamma \mathbf{p}\right)+m-\Sigma(p)$,

where $\Sigma(p)$ is a quark mass operator. In our problem, to consider the presence of the induced charge, we separate the part of radiation corrections $\Sigma^{(\text {tp. })}$ equaling to the sum of the tadpole diagrams with one gluon line $G_{4}^{3}$, which relates the quark bubble to a 
quark line. In Eq. (12), we also substitute the $A_{0}$ expression explicitly. In the rest frame of the plasma, where the actual calculations are carried out, the velocity vector is $u_{\mu}=\left(u_{4}=1, \mathbf{u}=0\right)$.

Next, we have to consider the gluon field propagator $G_{44}^{3}(k)$. For that, we use the generalized Green's function of neutral gluons. It reads (in the LorentzFeynman gauge) $[5,6]$

$\left(G_{44}^{3}\right)^{-1}=k^{2}-\Pi_{44}\left(k_{4}, \mathbf{k}\right)$,

where $\Pi_{44}\left(k^{2}\right)$ is the $4-4$ component of a polarization tensor. For $k_{4}=0, \mathbf{k} \rightarrow 0$, it defines Debye's temperature mass having the order $m_{D}^{2} \sim g^{2} T^{2}$. This mass is responsible for the screening of the Coulomb color fields.

The component of interest $G_{44}^{3}$ taken at zero momenta reads $[5,6]$

$G_{44}^{3}(p=0)=\frac{1}{m_{D}^{2}}$

Using the vertex of interactions in Eq. (12) and Eqs. (6), (14), we obtain

$\Sigma^{(\mathrm{tp} .)}=-\frac{\lambda^{3}}{2} \gamma^{4} \frac{g Q_{\mathrm{ind}}^{3}}{m_{D}^{2}}$.

Substituting this result in Eq. (12), we conclude that the resummation of tadpole insertions results in the replacement $g A_{0} \rightarrow g A_{0}+g \frac{Q_{\text {ind }}^{3}}{m_{D}^{2}}$ in the initial propagator.

\section{Potentials of Classical Color Fields}

The presence of the induced color charges in the plasma leads to the generation of classical gluon potentials. To describe this phenomenon, we introduce a simple model motivated by heavy-ion collisions. In this case, the plasma is created for a short period of time in a finite space volume which has a much smaller size in the direction of collisions compared to the transversal ones.

We consider the QGP confined in the plate of the size $L$ in the $z$-axis direction and infinite in the $x$-, $y$-directions. For this geometry, we calculate the classical potentials $\bar{\phi}^{3}=G_{4}^{3}, \bar{\phi}^{8}=G_{4}^{8}$ by solving the classical field equations for the gluon fields $G_{4}^{3}, G_{4}^{8}$ generated by the induced charges $Q_{\text {ind }}^{3}, Q_{\text {ind }}^{8}$. In doing so, we account for the results of Refs. $[5,6]$, where the gluon modes at the $A_{0}$ background were calculated. For our problem, we are interested in the longitudinal modes of the fields $G_{4}^{3}, G_{4}^{8}$ that have temperature masses $\sim g^{2} T^{2}$

The classical potential $\bar{\phi}^{3}$ is calculated from the equation

$\left[\frac{\partial^{2}}{\partial x_{\mu}^{2}}-m_{D}^{2}\right] \bar{\phi}^{3}=-Q_{\text {ind }}^{3}$

Making Fourier's transformation to the momentum $k$-space, we derive the spectrum of modes $-k_{4}^{2}=$ $=k_{x}^{2}+k_{y}^{2}+k_{z}^{2}+m_{D}^{2}$, where $k_{z}^{2}=\left(\frac{2 \pi}{L}\right)^{2} l^{2}$ and $l=$ $=0, \pm 1, \pm 2, \ldots$. The discreteness of $k_{z}$ is due to the periodic boundary condition for the plane: $\bar{\phi}^{3}(z)=$ $=\bar{\phi}^{3}(z+L)$. The general solution to Eq. (16) is

$\bar{\phi}^{3}\left(x_{4}, \mathbf{x}\right)=d+a e^{-i\left(k_{4} x_{4}-\mathbf{k} \mathbf{x}\right)}+b e^{i\left(k_{4} x_{4}-\mathbf{k} \mathbf{x}\right)}$.

In the case of zero induced charge, $d=0$, and we have two well-known plasmon modes. In the case of $Q_{\text {ind }}^{3} \neq$ $=0$, the values $a, b, d$ calculated from the confinement boundary condition

$\bar{\phi}^{3}\left(z=-\frac{L}{2}\right)=\bar{\phi}^{3}\left(z=\frac{L}{2}\right)=0$

result in the expression

$\bar{\phi}^{3}(z) \frac{Q_{\text {ind }}^{3}}{m_{D}^{2}}\left[1-\frac{\cos \left(k_{z} z\right)}{\cos \left(k_{z} L / 2\right)}\right]$.

The generated potential depends on the $z$-variable only. There are no dynamical plasmon states at all. The same result follows for the potential $\bar{\phi}^{8}(z)$. This is the main observation. In the presence of the induced charges, the static classical color potentials have to be realized in the plasma.

For applications, it is also necessary to get the Fourier transform $\bar{\phi}^{3}(k)$ of potential (19). Fulfilling that for the interval of $z\left[-\frac{L}{2}, \frac{L}{2}\right]$, we obtain

$\bar{\phi}^{3}(k)=\frac{Q_{\text {ind }}^{3} L}{m_{D}^{2}} \frac{\sin (k L / 2)}{(k L / 2)} \frac{k_{z}^{2}}{k_{z}^{2}-k^{2}}$,

where the values of $k_{z}$ are given by Eq. (16).

The energy for a mode with momentum $k_{z}$ is positive and equals

$E_{l}=\frac{\left(Q_{\text {ind }}^{3}\right)^{2}}{m_{D}^{4}} \frac{k_{z}^{2}}{2} L=\frac{\left(Q_{\text {ind }}^{3}\right)^{2}}{m_{D}^{4}} \frac{2 \pi^{2}}{L} l^{2}$.

ISSN 2071-0194. Ukr. J. Phys. 2019. Vol. 64, No. 8 
The total energy is given by the sum over $l$ of energies (21). Similar results hold for the potential $\bar{\phi}^{8}$.

Thus, in the presence of the induced charges, the static gluon potentials with positive energy should be generated. This is a consequence of condition Eq. (18). Obviously, such a situation is independent of the specific form of the bag, where the plasma is confined. In general, we have to expect that the color static potentials $\bar{\phi}^{3}, \bar{\phi}^{8}$ should be present in the QGP that results in a new type of processes.

\section{Effective $\gamma \gamma G$ vertices in QGP}

Other interesting objects, which have to be generated in QGP with the $A_{0}$ condensate, are the effective three-line vertices $\gamma \gamma G^{3}, \gamma \gamma G^{8}$. They also should exist due to Furry's theorem violation and relate the colored and white states. These vertices, in particular, lead to observable processes such as the inelastic scattering of photons, splitting (or conversion) of gluon $\bar{\phi}^{3}, \bar{\phi}^{8}$ potentials in two photons.

In this and next sections, we calculate the vertex $\gamma \gamma G^{3}$ and investigate the mentioned processes.

Let us consider the vertex $\Gamma_{\mu \lambda}^{\nu}$ corresponding to the diagram depicted in the plot. The second diagram is obtained by changing the direction of the quark line. We set that all the momenta are ingoing, the first photon is $\gamma_{1}\left(k_{\mu}^{1}\right)$, the second photon is $\gamma_{2}\left(k_{\lambda}^{3}\right)$, a color $a=3$ gluon $-Q^{3}\left(k_{\nu}^{2}\right)$, and $k^{1}+k^{2}+k^{3}=0$. $k^{1,2,3}$ are the momenta of external fields.

We consider the contributions coming from the traces of four $\gamma$-matrices, which are proportional to the quark mass and dominant for small photon momenta $k^{1}, k^{3} \ll m$. The analytic expression (common factor is $\left.e^{2} \mathrm{gm}\right)$ is

$\Gamma_{\mu \lambda}^{\nu}\left(k^{1}, k^{3}\right)=\Gamma_{\mu \lambda}^{\nu,(1)}\left(k^{1}, k^{3}\right)+\Gamma_{\mu \lambda}^{\nu,(2)}\left(k^{1}, k^{3}\right)$,

where

$$
\begin{aligned}
& \Gamma_{\mu \lambda}^{\nu,(1)}\left(k^{1}, k^{3}\right)= \\
& =\frac{1}{\beta} \sum_{p_{4}} \int \frac{d^{3} p}{(2 \pi)^{3}} \frac{N_{1}}{D(\tilde{P}) D\left(\tilde{P}-k^{1}\right) D\left(\tilde{P}+k^{3}\right)} .
\end{aligned}
$$

Here, the summation is over $p_{4}=\frac{2 \pi}{\beta}(l+1 / 2), l=0$, $\pm 1, \pm 2, \ldots$, the integration is over three-dimensional momentum space $p, N_{1}$ denotes the numerator coming from the first diagram, $\tilde{P}=\left(\tilde{P}_{4}=p_{4}-A_{0}, \mathbf{p}\right)$, $D(\tilde{P})=\left(p_{4}-A_{0}\right)^{2}+\mathbf{p}^{2}+m^{2}=\tilde{P}_{4}^{2}+\epsilon_{p}^{2}$, and $\epsilon_{p}^{2}=$

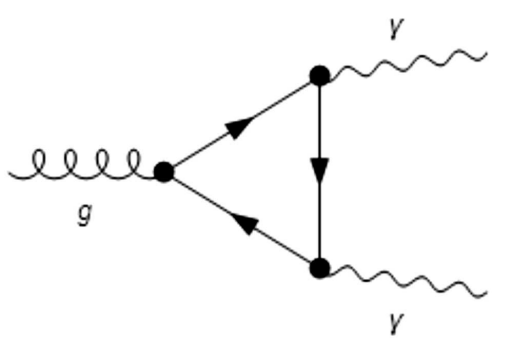

$=\mathbf{p}^{2}+m^{2}$ is the squared energy of a free quark. The functions $D\left(\tilde{P}-k^{1}\right), D\left(\tilde{P}+k^{3}\right)$ assume a corresponding shift in the momentum. The numerator $N_{1}$ is

$\left(N_{1}\right)_{\mu \nu \lambda}=\delta_{\mu \nu}\left(\tilde{P}-k^{2}\right)_{\lambda}+$

$+\delta_{\lambda \nu}\left(\tilde{P}-k^{2}\right)_{\mu}+\delta_{\mu \lambda}(\tilde{P}-q)_{\nu}$

where $q=k^{3}-k^{1}$ is the photon momentum transferred.

The expression for the second term in (22) comes from the second diagram and can be obtained from (23), (24) by the substitutions $k^{1} \rightarrow-k^{1}, k^{2} \rightarrow-k^{2}$, $q \rightarrow-q$. We denote the second numerator by $N_{2}$. In what follows, we carry out actual calculations for the first term in (22) and adduce the results for the second one.

Now, we consider the fact that, in the high temperature limit, the large values of the integration momentum $p$ give the leading contribution. Therefore, we can present the functions

$D(\tilde{P}), \quad D\left(\tilde{P}-k^{1}\right), \quad D\left(\tilde{P}+k^{3}\right)$

in the form:

$D(\tilde{P})=\tilde{P}_{4}^{2}+\epsilon_{p}^{2}=\tilde{P}^{2}$,

$D\left(\tilde{P}-k^{1}\right)=\tilde{P}^{2}\left(1-\frac{2 \tilde{P} k^{1}-k_{1}^{2}}{\tilde{P}^{2}}\right)$,

$D\left(\tilde{P}+k^{3}\right)=\tilde{P}^{2}\left(1+\frac{2 \tilde{P} k^{3}+k_{3}^{2}}{\tilde{P}^{2}}\right)$

with $k_{1}^{2}=\left(k_{4}^{1}\right)^{2}+\mathbf{k}_{1}^{2}, k_{3}^{2}=\left(k_{4}^{3}\right)^{2}+\mathbf{k}_{3}^{2}$. At high temperature and $\tilde{P}^{2} \rightarrow \infty$, the $k$-dependent terms are small. So, we can expand in these parameters. Now, the integrand in Eq. (23) reads

Intd. $=\frac{N_{1}}{\left(\tilde{P}^{2}\right)^{3}}\left[1+\sum_{i=1}^{4} A_{i}\right]$,

where

$A_{1}=-2 \frac{(\tilde{P} q)}{\tilde{P}^{2}}, \quad A_{2}=-\frac{k_{3}^{2}-k_{1}^{2}}{\tilde{P}^{2}}$, 
$A_{3}=-4 \frac{\left(\tilde{P} k^{1}\right)\left(\tilde{P} k^{3}\right)}{\tilde{P}^{2}}, \quad A_{4}=4 \frac{\left(\tilde{P} k^{1}\right)^{2}+\left(\tilde{P} k^{3}\right)^{2}}{\tilde{P}^{2}}$,

and the vector $q_{\mu}=\left(q_{4}, \mathbf{q}\right)$.

For the second diagram, we have to substitute $q \rightarrow$ $\rightarrow-q$, other terms are even and do not change.

Further, we concentrate on the scattering of photons on the potential $Q_{4}^{3}$ in the medium rest frame and set the thermostat velocity $u_{\nu}=(1, \mathbf{0}), \nu=4$. The corresponding terms in the numerators are

$N_{1} \rightarrow \delta_{\mu \lambda}(\tilde{P}+q)_{4}, \quad N_{2} \rightarrow \delta_{\mu \lambda}(\tilde{P}-q)_{4}$.

In this case, $\tilde{P}_{4}=p_{4}-A_{0}$ and $\tilde{P}^{2}=\left(p_{4}-A_{0}\right)^{2}+\epsilon_{p}^{2}$.

We have to calculate, in general, the series of two types corresponding to these numerators:

$S_{1}^{(n)}=\frac{1}{\beta} \sum_{p_{4}} \frac{p_{4}-A_{0}}{\left(\tilde{P}^{2}\right)^{n}}, \quad S_{2}^{(n)}=\frac{1}{\beta} \sum_{p_{4}} \frac{q_{4}}{\left(\tilde{P}^{2}\right)^{n}}$,

$n=3,4,5$.

These functions can be calculated from the $S_{1}^{(1)}$ and $S_{2}^{(1)}$, by computing a number of derivatives with respect to $\epsilon_{p}^{2}$. The latter series result in simple expressions. First is the one calculated already for the tadpole diagram Eq. (10). But now, we have to change the sign $A_{0} \rightarrow-A_{0}$. The function $S_{2}^{(1)}$ is

$S_{2}^{(1)}=\frac{1}{\beta} \sum_{p_{4}} \frac{q_{4}}{\tilde{P}^{2}}=-\frac{q_{4}}{2 \epsilon_{p}} \frac{\sinh \left(\epsilon_{p} \beta\right)}{\cos \left(A_{0} \beta\right)+\cosh \left(\epsilon_{p} \beta\right)}$.

Let us adduce the expressions for $A_{i}$ obtained after some simplifying algebraic transformations:

$$
\begin{aligned}
& A_{1}=-2 \frac{\left(p_{4}-A_{0}\right) q_{4}}{\tilde{P}^{2}} \\
& A_{3}=-\frac{4}{\tilde{P}^{2}}\left[\left(1-\frac{\epsilon_{p}^{2}}{\tilde{P}^{2}}\right) k_{4}^{1} k_{4}^{3}+\frac{\left(\mathbf{p k}_{1}\right)\left(\mathbf{p} \mathbf{k}_{3}\right)}{\tilde{P}^{2}}\right] \\
& A_{4}=\frac{4}{\tilde{P}^{2}}\left[\left(1-\frac{\epsilon_{p}^{2}}{\tilde{P}^{2}}\right)\left(\left(k_{4}^{1}\right)^{2}+\left(k_{4}^{3}\right)^{2}\right)+\right. \\
& \left.+\frac{\left(\mathbf{p} \mathbf{k}_{1}\right)^{2}+\left(\mathbf{p} \mathbf{k}_{3}\right)^{2}}{\tilde{P}^{2}}\right] .
\end{aligned}
$$

Finally, the resulting amplitude consists of the terms

$$
M_{1}=2 \delta_{\mu \lambda} \frac{p_{4}-A_{0}}{\left(\tilde{P}^{2}\right)^{3}}\left(1+A_{1}+A_{3}+A_{4}\right)
$$

and
$M_{2}=-4 \delta_{\mu \lambda} \frac{\left(p_{4}-A_{0}\right) q_{4}^{2}}{\left(\tilde{P}^{2}\right)^{4}}$.

Thus, all the contributions of the $S_{2}^{(n)}$ series are cancelled in the total. Now, we turn to the $d^{3} p$ integration.

We present calculation of high temperature asymptotic considering the first term in Eq. (34) which is calculated as the second derivative of $S_{1}^{(1)}$ over $\epsilon_{p}^{2}$ and equals to

$S_{3}=-A_{0} \beta \frac{\operatorname{sech}\left(\beta \epsilon_{p} / 2\right)^{4}}{64 p^{3}}\left(-2 \beta \epsilon_{p}+\right.$

$\left.+\beta \epsilon_{p} \cosh \left(\beta \epsilon_{p}\right)+\sinh \left(\beta \epsilon_{p}\right)\right)$.

Performing integration in the spherical coordinates and taking the leading order approximation, $\epsilon_{p} \beta=$ $=p \beta$, we get

$I_{3}=\int_{-\infty}^{\infty} d^{3} p S_{3}=-A_{0} \pi \beta$ (0.3348).

In such a way all the other integrations in Eqs. (34), (35) can be carried out.

\section{Scattering of Photons on the Potentials}

Relations (19), (20) give the calculated expressions for the potential $\bar{Q}_{4}^{3}=\bar{\phi}^{3}$ in the plasma plate. Here, we consider the scattering of photons on potential (20). Let us denote the momenta of ingoing and outgoing photons as $k_{\mu}^{1}$ and $k_{\lambda}^{3}$, respectively. The matrix element of the process is

$M=(2 \pi)^{4} \delta\left(k^{1}+k^{2}-k^{3}\right) \frac{e_{\mu}^{\sigma_{1}}}{\sqrt{2 \omega_{1}}} \bar{\phi}^{3} \Gamma_{\mu \lambda}^{4} \frac{e_{\lambda}^{\sigma_{3}}}{\sqrt{2 \omega_{3}}}$.

Here, $e^{\sigma_{1}}{ }_{\mu}, e^{\sigma_{2}}{ }_{\lambda}$ are polarization amplitudes of photons, and $\omega_{1}, \omega_{3}$ are the corresponding energies, $\Gamma_{\mu \lambda}^{4}\left(k^{1}, k^{3}\right)$ is the effective vertex calculated in the previous section.

We assume that the beams are not polarized, $\sum_{\sigma_{3}} e_{\mu}^{\sigma_{1}} e_{\mu^{\prime}}^{\sigma_{1}}==\delta_{\mu \mu^{\prime}}, \quad \sum_{\sigma_{3}} e_{\lambda^{2}}^{\sigma_{3}} e_{\lambda^{\prime}}^{\sigma_{3}}=\delta_{\lambda \lambda^{\prime}}$. Then the probability

$P=M M^{+}=\left(\bar{\phi}^{3}(k)\right)^{2} \Gamma_{\mu \lambda}^{4} \Gamma_{\mu \lambda}^{4} \frac{C}{4 \omega_{1} \omega_{3}} \delta\left(k^{1}+k^{2}-k^{3}\right)$,

where $C$ is some nonrelevant number. In this expression (accounting for the momentum conservation),

ISSN 2071-0194. Ukr. J. Phys. 2019. Vol. 64, No. 8 
$\omega_{3}=\left[\left(\omega_{x}^{1}\right)^{2}+\left(\omega_{y}^{1}\right)^{2}+\left(\omega_{z}^{1}+k_{z}^{2}\right)^{2}\right]^{1 / 2}$. The value of $k_{z}^{2}$ is a free parameter of the problem. It indicates the point, at which the actual scattering happens in the z-plane. Since this is not known, we have to sum up the probability over $k_{z}^{2}$, i.e., over $l$. In this expression, all the parameters and functions are known. So, the scattering on the induced color potentials can be calculated. Analogous process has to happen for the classical field $\bar{\phi}^{8}(k)$. This kind of scattering drastically differs from that for the plasma consisting of free chaotically moving particles.

Another related process is the conversion of classical gluon fields $\bar{\phi}^{3}(k), \bar{\phi}^{8}(k)$ in two photons coming out from the QGP due to the effective vertex $\Gamma_{\mu \lambda}^{\nu}\left(k^{1}, k^{3}\right)$. In the rest frame of the plasma, two photons moving in opposite directions and having specific energies, which correspond to the energy levels $E_{l}$ Eq. (21), have to be observed. The amplitude is described by Eq. (38) with corresponding changes of momenta.

\section{Conclusions}

We have demonstrated that, in QGP with the $A_{0}$ condensates, the induced color charges $Q_{\text {ind }}^{3}, Q_{\text {ind }}^{8}$ and the static classical gluon fields $\bar{\phi}^{3}, \bar{\phi}^{8}$ have to be present. This results in specific new phenomena. In particular, the conversion of gluons in photons happened due to the effective $\Gamma_{\mu \lambda}^{\nu}$ vertex could influence the exit of direct photons from the plasma.

1. Y. Aoki, et al. The QCD transition temperature: results with physical masses in the continuum limit II. arxiV: 0903.4155 (2009).
2. H. Satz. Extreme states of matter in strong interaction physics: An introduction. Lect. Notes Phys. 841, 237 (2012).

3. M. Srednicki, I. Susskin. Colored monopoles on the lattice. Nucl. Phys. B 179, 239 (1981).

4. O.A. Borisenko, J. Bohacik, V.V. Skalozub. $A_{0}$ condensate in QCD. Fortschr. Phys. 43, 301 (1995).

5. O.K. Kalashnikov. Infrared properties of the hot gauge theory after symmetry breaking. Progr. Theor. Phys. 92, 1207 (1994).

6. O.K. Kalashnikov. Self-energy peculiarities of the hot gauge theory after symmetry breaking. Mod. Phys. Lett. A 11, 1825 (1996).

7. I.A. Baranov, V.V. Skalozub. Induced charges and quark polarization operator at A0 background. J. Phys. Electron. 26 (1), 3 (2018).

Received 26.07.19

\section{В. Скалозуб}

ІНДУКОВАНІ КОЛЬОРОВІ ЗАРЯДИ, ЕФЕКТИВНА $\gamma \gamma G$-ВЕРШИНА У КВАРК-ГЛЮОННІЙ ПЛАЗМІ. ЗАСТОСУВАННЯ ДО ЗІТКНЕНЬ ВАЖКИХ IOHIВ

$\mathrm{P}$ е $з$ ю м е

Ми обчислюємо індуковані кольорові заряди $Q_{\text {ind }}^{3}, Q_{\text {ind }}^{8}$ та ефективну $\gamma-\gamma$-глюон вершину, які генеруються у кваркглюонній плазмі в присутності $A_{0}$ конденсату внаслідок порушення кольорової С-парності в таких умовах. Для імітації зіткнення важких ядер ми розглядаємо модель плазми, що знаходиться всередені вузької пластини необмежених поперечних розмірів. Для таких умов ми отримуємо потенціали класичних глюонних полів $\bar{\phi}^{3}, \bar{\phi}^{8}$, що виникають у присутності індукованих зарядів. У якості застосування розглядаються два процеси - розсіювання фотонів на плазмі та конвертація класичних глюонів у два фотони, що випромінюються із плазми. 